\title{
Cambios en los parámetros lipídicos en pacientes sometidos a cirugía bariátrica. Parámetros lipídicos y cirugía bariátrica
}

\author{
Changes in lipid parameters in patients undergoing bariatric \\ surgery. Lipid parameters and bariatric surgery \\ Gabriela Ruiz-Mar, ${ }^{*}$ Luis Alfredo Ornelas-Oñate, ${ }^{\ddagger}$ \\ Alondra Ruelas-Ayala, ${ }^{*}$ Jorge Enrique Ramírez-Velásquez ${ }^{\S}$
}

Palabras clave: obesidad, cirugía bariátrica, índice aterogénico, colesterol, triglicéridos, colesterol no-HDL.

Keywords: Obesity, bariatric surgery, atherogenic index, cholesterol, triglycerides, non-HDL cholesterol.
* Residente de Alta Especialidad en Cirugía Bariátrica. ¥ Cirujano Bariatra. $\S$ Jefe de Servicio de Cirugía Bariátrica.

Servicio de Cirugía Bariátrica, Hospital General de México.

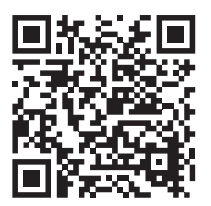

\section{RESUMEN}

Introducción: La obesidad se asocia a múltiples comorbilidades como la dislipidemia. Objetivo: Analizar los cambios en los parámetros lipídicos en pacientes sometidos a procedimientos de cirugía bariátrica. Material y métodos: Estudio longitudinal en pacientes sometidos a cirugía bariátrica en 2016. Se registraron los cambios posteriores al procedimiento. Resultados: Se incluyeron 66 pacientes. Se realizó manga gástrica en $33.3 \%$, bypass en Y de Roux en $22.7 \%$ y bypass de una anastomosis en $43.9 \%$. Peso inicial de $122 \pm 26.9 \mathrm{~kg}$ e IMC inicial de $46.72 \pm 8.7 \mathrm{~kg} / \mathrm{m}^{2}$. Se presentó dislipidemia aterogénica (DA) en $83.3 \%$. Se encontraron diferencias significativas en todas las variables estudiadas al año de seguimiento; sin diferencia entre las diferentes técnicas en peso: $\mathrm{p}=0.674, \% \mathrm{EPP}: \mathrm{p}=0.420$, TGC: $p=0.287$ y c-HDL: $p=0.432$; si en los cambios de CT $(p=0.0001)$ y c-LDL $(p=0.01)$. Al evaluar los índices, en todos se encontró diferencia significativa al inicio y a los 12 meses de seguimiento $(\mathrm{p}=0.001)$ sin diferencia entre las técnicas estudiadas. Con respecto a la DA, se observó mejoría significativa al año de seguimiento $(\mathrm{p}=0.05)$ con remisión en $74 \%$. Conclusiones: La cirugía bariátrica se asocia a mejoría de los diferentes parámetros lipídicos con remisión de la DA en la mayoría de los pacientes independientemente del tipo de procedimiento realizado.
Introduction: Obesity is associated with multiple comorbidities such as dyslipidemia. Objective: To analyze the changes in lipid parameters in patients undergoing bariatric surgery procedures. Material and methods: Longitudinal study in patients undergoing bariatric surgery in 2016. Post-procedure changes were recorded. Results: 66 patients were included. Gastric sleeve was performed in $33.3 \%$, Roux-en-Y gastric bypass (RYGB) in $22.7 \%$ and an anastomosis bypass in 43.9\%. Initial weight of $122 \pm$ $26.9 \mathrm{~kg}$ and initial BMI of $46.72 \pm 8.7 \mathrm{~kg} / \mathrm{m} 2$. Atherogenic dyslipidemia (AD) occurred in $83.3 \%$. Significant differences were found in all the variables studied at the year of follow-up; no difference between the different techniques in weight: $p=0.674, \% E B W L: p=0.420$, TGC: $p=0.287$ and $c-H D L: p=0.432$; if in the changes of CT $(p=0.0001)$ and $c-L D L(p=0.01)$. When evaluating the indexes, a significant difference was found in all at the beginning and at 12 months of follow-up ( $p=0.001$ ); no difference between the techniques studied. With respect to $A D$, significant improvement was observed at one year follow-up $(p=0.05)$ with remission in $74 \%$. Conclusions: Bariatric surgery is associated with improvement of the different lipid parameters, with remission of $A D$ in most patients regardless of the type of procedure performed.

\section{INTRODUCCIÓN}

$\mathrm{E}$ I sobrepeso y la obesidad se definen como Una acumulación anormal o excesiva de grasa que es perjudicial para la salud. En adultos, la Organización Mundial de la Salud (OMS) define el sobrepeso y la obesidad como: 1) sobrepeso cuando el IMC (índice de masa corporal) es de $25-29.9 \mathrm{~kg} / \mathrm{m}^{2} ; 2$ ) obesidad como un IMC igual o superior a $30 \mathrm{~kg} / \mathrm{m}^{2}$. Según los datos de la OMS en 2014 más de 1,900 millones de adultos de 18 o más años tenían sobrepeso, de los cuales, más

Citar como: Ruiz-Mar G, Ornelas-Oñate LA, Ruelas-Ayala A, Ramírez-Velásquez JE. Cambios en los parámetros lipídicos en pacientes sometidos a cirugía bariátrica. Parámetros lipídicos y cirugía bariátrica. Cir Gen. 2020; 42 (3): 203-209 https://dx.doi.org/10.35366/99960 
de 600 millones eran obesos. ${ }^{1}$ Acorde a datos de la Encuesta Nacional de Salud y Nutrición (ENSANUT) 2016, tres de cada 10 menores de 11 años padecen sobrepeso u obesidad así como cuatro de cada 10 adolescentes y siete de cada 10 adultos; se observó un aumento en las cifras de sobrepeso y obesidad en mujeres adultas y en las zonas rurales. ${ }^{2}$

La obesidad está asociada a la presencia de resistencia a la insulina en tejidos periféricos junto con la existencia de un estado proinflamatorio causado principalmente por la liberación de distintas citocinas y hormonas por parte del tejido adiposo. ${ }^{3}$ Se ha relacionado la obesidad con la presencia de dislipidemia aterogénica, caracterizada por bajos niveles de colesterol de alta densidad (c-HDL), hipertrigliceridemia y aumento en los niveles de colesterol de baja densidad (c-LDL). Por otro lado, la cirugía bariátrica ha demostrado ser una alternativa de tratamiento eficaz en pacientes con obesidad y sus comorbilidades. Se realiza el presente trabajo para valorar los cambios en los diferentes índices lipídicos posterior a la cirugía bariátrica.

\section{MATERIAL Y MÉTODOS}

Se llevó a cabo un estudio observacional, longitudinal y analítico aprobado por el co- mité de investigación de la institución con número de registro de DIR/19/310B/3/006, donde se evaluaron los pacientes sometidos a cirugía bariátrica en el Centro de Atención Integral de Diabetes y Obesidad (CAIDO) del Hospital General de México en el periodo comprendido de enero a diciembre de 2016, se excluyeron los pacientes que consumían fármacos hipolipemiantes o que presentaran datos de síndrome metabólico. La indicación del tipo de procedimiento quirúrgico (manga gástrica, bypass gástrico en $\mathrm{Y}$ de Roux o bypass gástrico de una anastomosis) se basó en los criterios clínicos y el consenso de los diferentes especialistas de la clínica. Todos los pacientes fueron evaluados en el preoperatorio y a uno, tres, seis y 12 meses postoperatorios; durante cada visita se valoró peso, IMC, \% exceso de peso perdido (\%EPP) y pruebas bioquímicas de colesterol total $(\mathrm{CT})$, c-HDL, C-LDL y triglicéridos (TGC).

El índice de masa corporal (IMC) se calcula como el peso en kilogramos entre la talla al cuadrado $\left(\mathrm{kg} / \mathrm{m}^{2}\right) ;{ }^{1}$ el \%EPP se calculó basado en el exceso de peso comparado con el peso correspondiente a un IMC $25 \mathrm{~kg} / \mathrm{m}^{2}$ en cada paciente. ${ }^{4} \mathrm{El}$ índice aterogénico (IA) se obtuvo con la relación $\mathrm{CT} /$ colesterol-HDL

\begin{tabular}{lcccc}
\multicolumn{5}{c}{ Tabla 1: Características basales de los pacientes. } \\
& Manga (22) & BAGUA (29) & BGYR (15) & p \\
\hline Peso (kg) & $125 \pm 26.0$ & $122 \pm 33.0$ & $118 \pm 29$ & 0.796 \\
IMC (kg/m²) & $47 \pm 9.0$ & $47 \pm 9.0$ & $45 \pm 8$ & 0.799 \\
CT (mg/dl) & $180 \pm 34.0$ & $171 \pm 33.0$ & $178 \pm 35$ & 0.663 \\
TGC (mg/dl) & $167 \pm 97.0$ & $154 \pm 62.0$ & $203 \pm 107$ & 0.199 \\
c-HDL (mg/dl) & $38.6 \pm 10.0$ & $39.8 \pm 9.0$ & $38.2 \pm 10$ & 0.837 \\
& & & \\
c-LDL (mg/dl) & $106.6 \pm 28.0$ & $106.5 \pm 23.0$ & $105.3 \pm 35$ & 0.988 \\
CT/cHDL & $4.9 \pm 1.2$ & $4.5 \pm 1.1$ & $4.9 \pm 1.3$ & 0.378 \\
TGC/cHDL & $4.7 \pm 3.5$ & $4.03 \pm 1.8$ & $5.5 \pm 2.7$ & 0.210 \\
c-LDL/c-HDL & $2.9 \pm 0.9$ & $2.8 \pm 0.8$ & $2.9 \pm 1.1$ & 0.860 \\
No HDL & $140.9 \pm 33.0$ & $131 \pm 36.0$ & $139.3 \pm 35.0$ & 0.555 \\
\hline BAGUA = bypass gástrico de una anastomosis, BGYR = bypass gástrico en Y de Roux, IMC = índice de masa \\
corporal, CT = colesterol total, TGC = triglicéridos, c-HDL = colesterol de alta densidad, c-LDL = colesterol de baja \\
densidad. \\
Elaboración propia.
\end{tabular}


Tabla 2: Evolución de los diferentes parámetros lipídicos.

\begin{tabular}{|c|c|c|c|c|c|c|c|}
\hline & \multicolumn{2}{|c|}{ Manga } & \multicolumn{2}{|c|}{ BAGUA } & \multicolumn{2}{|c|}{ BGYR } & \multirow[b]{2}{*}{$\mathrm{p}^{*}$} \\
\hline & Inicio & 12 meses & Inicio & 12 meses & Inicio & 12 meses & \\
\hline CT/cHDL & $4.9 \pm 1.2$ & $3.5 \pm 0.8$ & $4.5 \pm 1.1$ & $3.3 \pm 0.9$ & $4.9 \pm 1.3$ & $3.3 \pm 1.1$ & 0.672 \\
\hline TGC/cHDL & $4.7 \pm 3.5$ & $2.1 \pm 0.8$ & $4.03 \pm 1.8$ & $2.3 \pm 1.2$ & $5.5 \pm 2.7$ & $2.3 \pm 1.2$ & 0.23 \\
\hline c-LDL/c-HDL & $2.9 \pm 0.9$ & $2 \pm 0.6$ & $2.8 \pm 0.8$ & $1.9 \pm 0.6$ & $2.9 \pm 1.1$ & $1.8 \pm 0.9$ & 0.77 \\
\hline No HDL & $140.9 \pm 33.0$ & $122 \pm 23.0$ & $131 \pm 36.0$ & $102 \pm 25.0$ & $139.3 \pm 35.0$ & $104 \pm 28.0$ & 0.07 \\
\hline Dislipidemia (\%) & 19 (86.4) & $4(18.2)$ & 23 (79.3) & $8(27.6)$ & 13 (86.7) & $3(20.0)$ & 0.05 \\
\hline
\end{tabular}

considerando como predictor de riesgo coronario un valor $>4.5 ;^{5}$ el índice TGC/c-HDL se obtuvo dividiendo los triglicéridos totales en ayuno entre el total de c-HDL, donde los valores de corte que se han asociado a cifras elevadas de insulina (como indicador de resistencia a la insulina) corresponden a 2.5 en mujeres y a 3.5 en hombres, ${ }^{6}$ por lo que se ha estandarizado un valor mayor de tres en adultos como marcador de insulinorresistencia; $;^{7,8}$ el colesterol no HDL (no-HDL) es una medida del colesterol LDL, de densidad intermedia (IDL) y de muy baja densidad $(\mathrm{VLDL})^{5}$, se calcula restando el colesterol HDL al valor de colesterol total, se ha encontrado que el colesterol no-HDL es el principal determinante de aterosclerosis precoz. ${ }^{9}$ Se definió dislipidemia aterogénica (DA) como un valor de triglicéridos $>150 \mathrm{mg} / \mathrm{dl}$ y c-HDL menor de $40 \mathrm{mg} / \mathrm{dl}$ hombres y $50 \mathrm{mg} / \mathrm{dl}$ mujeres. ${ }^{4}$

Se realizó un cálculo de tamaño de muestra con el programa Gpower 3.1 con un total de 40 pacientes para un poder estadístico de 0.8 , error a de 0.05 y error $b$ de 0.2. Para el análisis de datos se utilizaron medidas de tendencia central como estadística descriptiva, modelo lineal general de medidas repetidas para evaluar los cambios en los parámetros de laboratorio y pérdida de peso y análisis de ANOVA para evaluar las diferencias entre las diversas técnicas. Se consideró un valor de $\mathrm{p}<0.05$ como estadísticamente significativo. Se llevó a cabo el análisis en IBM SPSS V20.

\section{RESULTADOS}

Se estudió un total de 66 pacientes. El grupo estuvo conformado por 56 mujeres (84.4\%) y 10 hombres (15.6\%). Los procedimientos quirúrgicos realizados fueron: 22 mangas gástricas (33.3\%), 15 bypass en Y de Roux (22.7\%) y 29 bypass de una anastomosis (43.9\%). Las características basales de los pacientes se muestran en la Tabla 1.

Se realizó un ANOVA de medidas repetidas para evaluar los cambios de las diferentes variables en el tiempo (Figura 1). Se encontraron diferencias estadísticamente significativas en todas las variables estudiadas al año de seguimiento; no se observó diferencia en el peso $(p=0.674), \%$ EPP $(p=0.420)$, TGC $(p=0.287)$ y $c-H D L(p=0.432)$ entre las diferentes técnicas; se apreció diferencia estadística en los cambios de CT $(p=0.0001)$ y c-LDL $(p=0.01)$.

Al evaluar los diferentes índices, en todos se encontró diferencia estadísticamente significativa al inicio y a los 12 meses de seguimiento $(p=0.001)$ (Figura 2); no se detectó diferencia entre las técnicas estudiadas (Tabla 2). Con respecto a la DA, se presentó en $83.3 \%$ de los pacientes al ingreso; 19 (86.3\%) en manga gástrica, 23 (79.3\%) en BAGUA y 13 (86.6\%) en BGYR. Se observó mejoría estadísticamente significativa al año de seguimiento $(p=0.05)$ con remisión de la misma en $74 \%$ de los pacientes, sin diferencia entre las diferentes técnicas. 


\section{DISCUSIÓN}

La obesidad se ha convertido en una pandemia que se considera un tema prioritario para la salud, la investigación y la economía en el mundo. La prevalencia de la DA está asociada a sobrepeso, obesidad, diabetes mellitus e infarto al miocardio. ${ }^{10}$ En el presente estudio se observó que $83.3 \%$ de los pacientes mostraron criterios para DA previo a la cirugía, con remisión de la misma en $74 \%$ de los casos posterior al procedimiento de cirugía

Figura 1:

Evolución de las diferentes variables posterior a la cirugía bariátrica. Todas las variables presentan mejoría estadísticamente significativa a los 12 meses de la intervención independientemente del tipo de procedimiento quirúrgico utilizado $(p<0.05)$. El valor de p mostrado en la imagen representa diferencia entre las distintas técnicas para cada variable: A) peso $p=0.674$; B) $\% E P P p=$ 0.420 ;C) colesterol total $p=0.001 ; \boldsymbol{D}$ ) triglicéridos $p=$ 0.237; E) c-HDL $p=0.432 ; \boldsymbol{F})$ $c-L D L p=0.01$. \%ЕPP: \% exceso de peso perdido. Los resultados mostrados son la media de cada variable.

Elaboración propia.
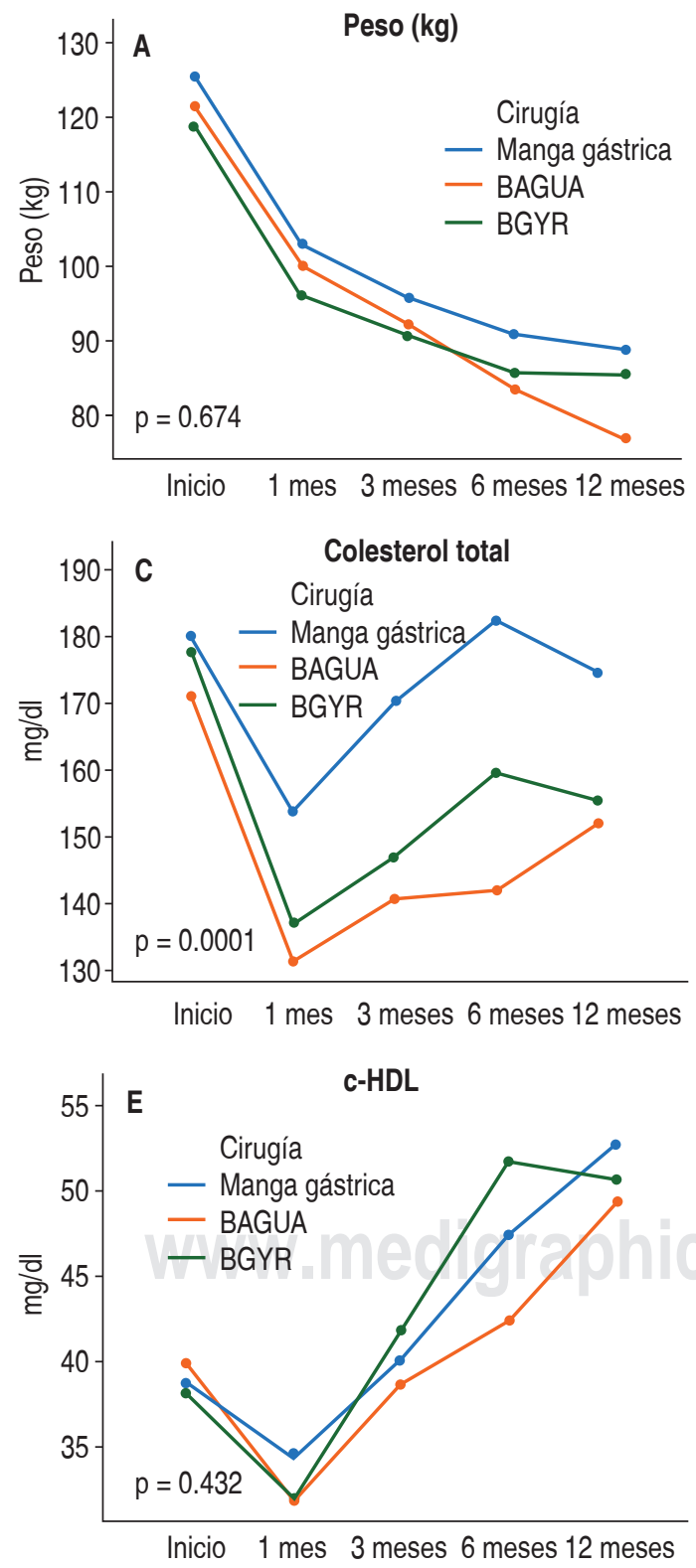
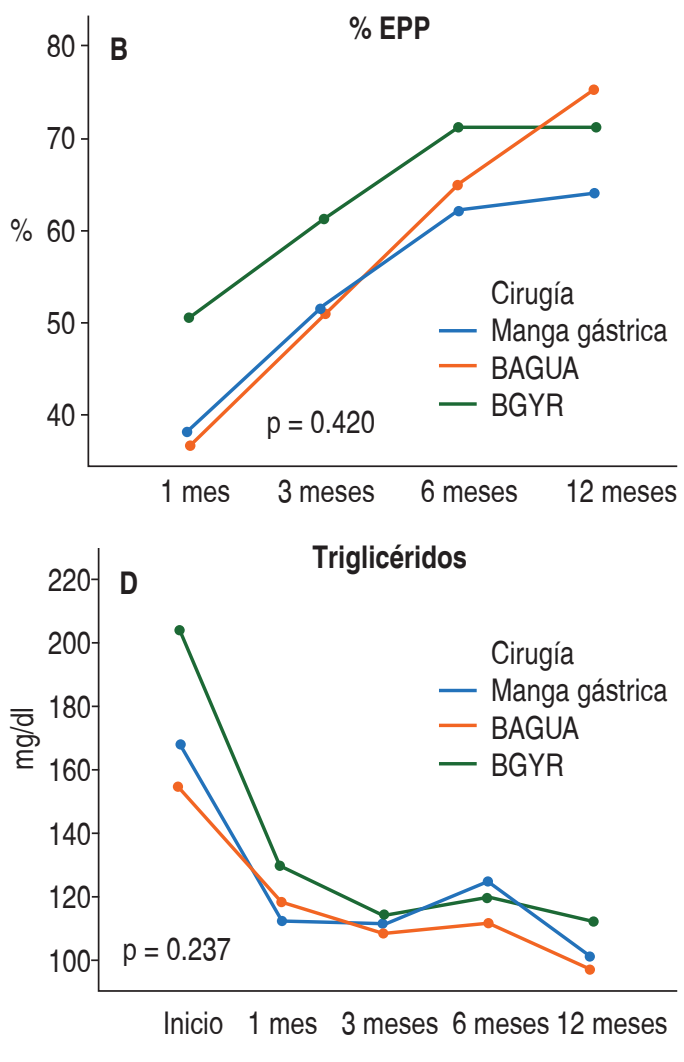

Inicio 1 mes 3 meses 6 meses 12 meses

bariátrica, esto se correlaciona con los datos reportados previamente, donde $20-63 \%$ de los pacientes prequirúrgicos presentaban criterios de DA con una remisión posterior a la cirugía de $62-74 \% .4,11$ No se encontraron diferencias significativas en la remisión entre los pacientes sometidos a manga gástrica, bypass en $\mathrm{Y}$ de Roux o bypass de una anastomosis como se ha reportado en la literatura. ${ }^{12,13}$ Asimismo, se observó una resolución de los niveles de TGC a rangos normales en $89.4 \%$ de los pacientes, de CT en $89.3 \%$ y de c-HDL en $74.2 \%$ a los

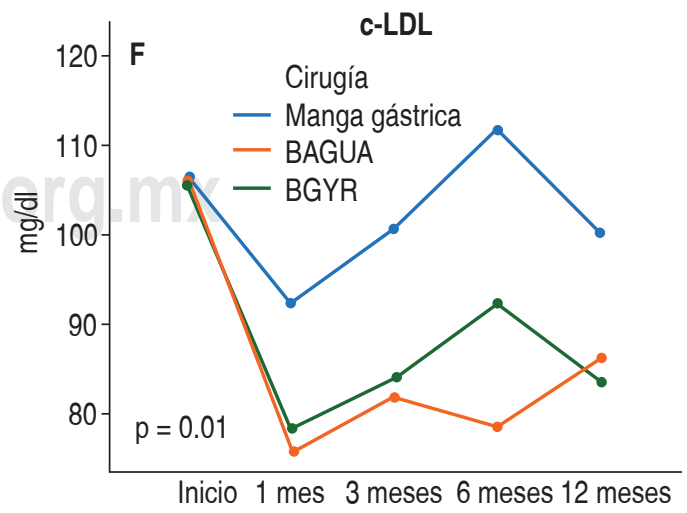




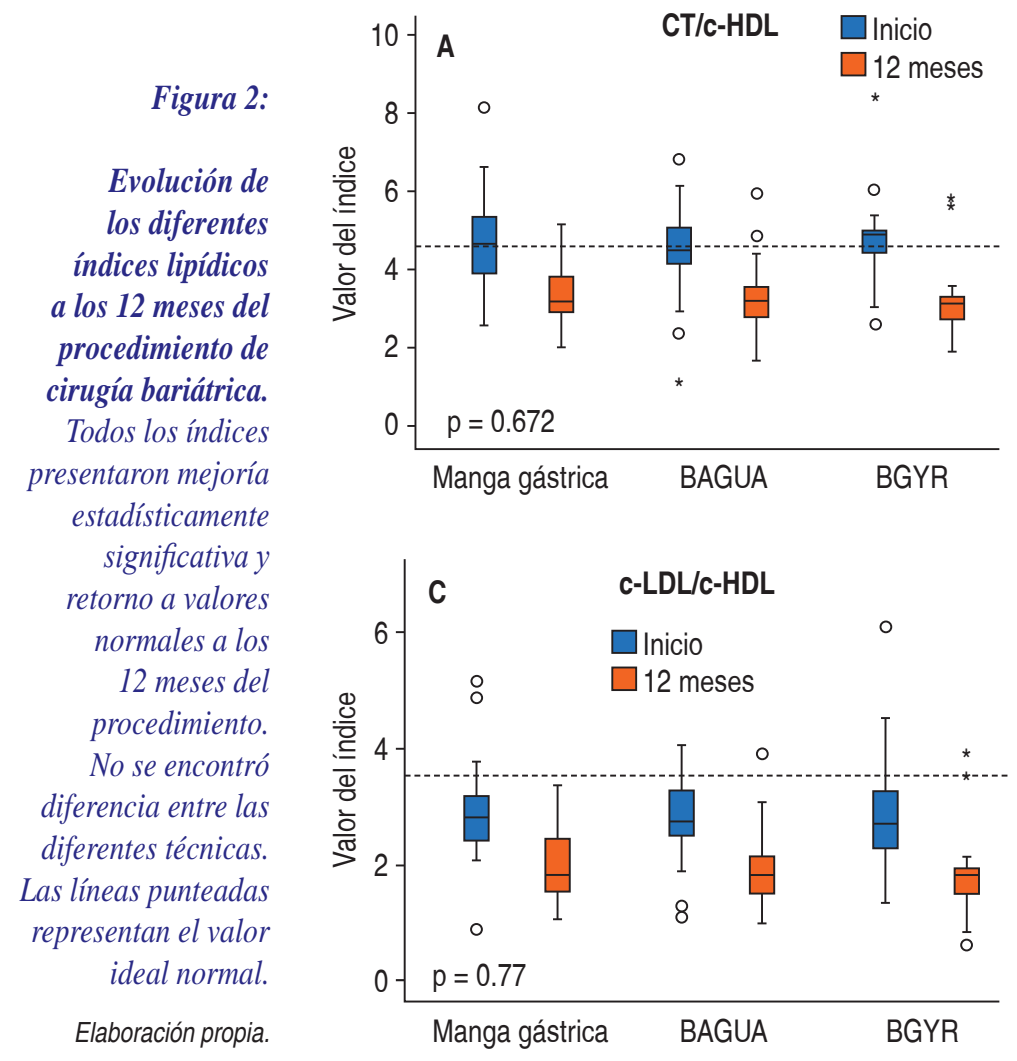

12 meses de seguimiento, lo cual concuerda con lo reportado en estudios previos, donde se presenta una disminución de $30-63 \%$ en TGC y un aumento de $12-39 \%$ posterior a la cirugía bariátrica. ${ }^{14,15}$

Los valores de CT disminuyeron a valores normales hasta en $89.3 \%$ de los casos, lo cual se correlaciona con un aumento del c-HDL y disminución del c-LDL, siendo de mayor importancia el grado de aumento de c-HDL para valorar la disminución de riesgo cardiovascular. ${ }^{15}$

Con respecto al c-HDL, es importante destacar que se observó una disminución abrupta de sus valores en el primer mes posterior al procedimiento quirúrgico, probablemente debido a la dieta posterior al mismo seguido de un aumento franco y estadísticamente significativo a partir de los tres meses postquirúrgicos, correlacionando con descripciones previas en la literatura son independientes del tipo de cirugía empleada, ${ }^{12,16}$ lo cual sugiere que el aumento de los valores de c-HDL sí tiene una correlación directa con la pérdida de peso posterior al procedimiento.
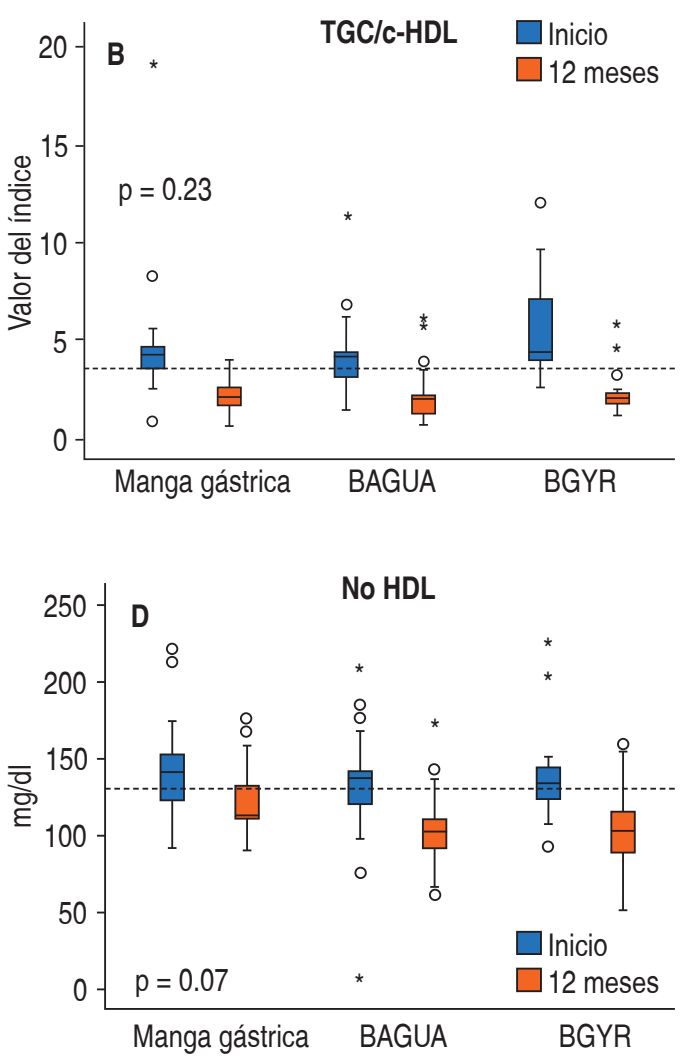

El colesterol no-HDL se ha recomendado como una meta terapéutica en los pacientes que presentan hipertrigliceridemia y alto riesgo cardiovascular y se ha sugerido que puede ser un marcador de la concentración de apoB en la práctica clínica. ${ }^{5}$ En este estudio se observó una disminución en los niveles de colesterol no-HDL aunado al aumento en los niveles de c-HDL con una disminución menos marcada en los niveles de CT como se ha reportado previamente. ${ }^{15}$

El IA se ha relacionado a resistencia a la insulina y como el mejor predictor de riesgo cardiovascular. ${ }^{5}$ Se detectó la mayor disminución del nivel del índice aterogénico a los tres meses postquirúrgicos, manteniéndose durante el seguimiento igual que lo reportado por Climent y colaboradores, ${ }^{4}$ quienes informaron que la disminución en los niveles del IA se correlaciona con una remisión de la DA y una mejoría en el índice HOMA-IR.

Al comparar nuestros resultados con otros centros de atención a la obesidad y sus comorbilidades en México observamos que 
Molina-Ayala y colaboradores ${ }^{17}$ informaron una disminución significativa en el peso, glucosa, insulina, HOMA-IR, CT, TGC, c-HDL, c-LDL y ácido úrico un año posterior al procedimiento de cirugía bariátrica. Reyes-Pérez y su equipo ${ }^{18}$ mostraron resultados similares en pacientes postoperados de bypass gástrico en $\mathrm{Y}$ de Roux en un medio privado. La edad de los pacientes fue de $40.1 \pm 11.5$ años, las mujeres representaron $42 \%$ del grupo y el IMC inicial fue de 42 $\pm 6.5 \mathrm{~kg} / \mathrm{m}^{2}$. Asimismo, en el Instituto Nacional de Ciencias Médicas y Nutrición "Salvador Zubirán" se analizaron pacientes postoperados de bypass gástrico en $\mathrm{Y}$ de Roux encontrando una edad promedio de $38 \pm 10$; el IMC al inicio del estudio se observó en $48 \pm 6 \mathrm{~kg} / \mathrm{m}^{2}$ con un $\%$ EPP de $70 \%$ al año del procedimiento. Los valores de c-HDL, c-LDL y TGC disminuyeron significativamente al año del procedimiento quirúrgico. ${ }^{19}$

La reducción en los diferentes parámetros lipídicos posterior a la cirugía bariátrica se ha asociado a una disminución en los eventos cardiovasculares y en las muertes por la misma causa, ${ }^{20}$ por lo que los resultados en la remisión y mejoría de las diferentes alteraciones lipídicas en los pacientes sometidos a cirugía bariátrica son de suma importancia. Dentro de las limitantes en el presente estudio encontramos que los datos de estilo de vida como hábitos alimenticios y actividad física no fueron valorados y al contar con un tamaño de muestra reducido es difícil extrapolar los resultados con una adecuada potencia estadística.

\section{CONCLUSIONES}

La cirugía bariátrica se asocia a la mejoría franca de los diferentes parámetros lipídicos, con remisión de la DA en la mayoría de los pacientes a partir del tercer mes y continuando a 12 meses posteriores a la cirugía $(p=0.0001)$. La pérdida de peso parece tener el mayor impacto en la disminución de los diferentes índices y esta mejoría es independiente del tipo de procedimiento que se realice; peso $(p=0.674)$, $\% \operatorname{EPP}(p=0.420)$, TGC $(p=0.287)$ y $c-H D L$ $(p=0.432)$ entre las diferentes técnicas. Por lo anterior, podemos concluir que la cirugía bariátrica es una opción terapéutica en los pacientes obesos con DA desde los primeros meses del procedimiento. Al ser éste un estudio preliminar, debemos continuar observando el comportamiento a mayor tiempo y con un número más amplio de pacientes.

\section{REFERENCIAS}

1. Organización Mundial de la Salud (Internet). México; 2018 (Citado 3 de feb 2018). Obesidad y sobrepeso. Disponible en: http://www.who.int/mediacentre/ factsheets/fs311/es/.

2. Instituto Nacional de Salud Pública (Internet). México; 2018 (Citado 3 de feb 2018). Encuesta Nacional de Salud y Nutrición de Medio Camino 2016 Informe Final de Resultados. Disponible en: http://oment.uanl. mx/wp-content/uploads/2016/12/ensanut_mc_2016310oct.pdf.

3. Martinez J, Torres P, Juárez M. Los ácidos grasos y la lipotoxicidad: implicaciones metabólicas. Rev Fac Med. 2013; 56: 5-18.

4. Climent E, Benaiges D, Pedro-Botet J, Flores-Le Roux J, Ramón J, Villatoro M, et al. Atherogenic dyslipidemia remission 1 year after bariatric surgery. Obes Surg. 2017; 27: 1548.

5. Millán J, Pintó X, Muñoz A, Zúñiga M, Rubiés-Prat J, Pallardo LF, et al. Lipoprotein ratios: physiological significance and clinical usefulness in cardiovascular prevention. Vasc Health Risk Manag. 2009; 5: 757 765.

6. Hernández-Vite Y, Elizalde-Barrera C I, FloresAlcántar M G, Vargas Ayala G, Loreto-Bernal M. Asociación entre el índice triglicéridos/colesterol HDL y la glucosa alterada en ayuno en pacientes normotensos con obesidad y sobrepeso. Med Int Méx. 2015; 31: 507-515.

7. Soutelo J, Graffigna $M$, Honf $M$, Migliano $M$, Aranguren $M$, Proietti $A$, et al. Índice triglicéridos/ HDL-colesterol: en una población de adolescentes sin factores de riesgo cardiovascular. Alan. 2012; 62: 167-71.

8. González-Chávez A, Simental-Mendía L E, ElizondoArgueta S. Relación triglicéridos/colesterol-HDL elevada y resistencia a la insulina. Cir Cir. 2011; 79: 126-131.

9. Acevedo M, Kramer V, Tagle R, Coebalan R, Arnaíz $\mathrm{P}$, Berrios $\mathrm{X}$, et al. Relación colesterol total a HDL y colesterol no HDL: los mejores indicadores lipídicos de aumento de grosor de la íntima media carotidea. Rev Med Chile. 2012; 140: 969-976.

10. Pedro-Botet J, Flores-Le Roux JA, Mostaza JM, Pintó X, de la Cruz JJ, Banegas JR, et al. Dislipemia aterogénica: prevalencia y control en las unidades de lípidos. Rev Clin Esp. 2014; 214: 491-498.

11. Courcoulas AP, Christian NJ, Belle SH, Berk PD, Flum DR, Garcia L, et al. For the longitudinal assessment of bariatric surgery (LABS) consortium. Weight change and health outcomes at 3 years after bariatric surgery among individuals with severe obesity. JAMA. 2013; 310: 2416-2425.

12. Benaiges D, Flores-Le-Roux JA, Pedro-Botet J, Ramon JM, Parri A, Villatoro $M$, et al. Impact of restrictive (sleeve gastrectomy) vs hybrid bariatric surgery (Roux- 
en-Y gastric bypass) on lipid profile. Obes Surg. 2012; 22: 1268-1275.

13. Vidal P, Ramón J, Goday A, Benaiges D, Trillo L, Parri A et al. Laparoscopic gastric bypass versus laparoscopic sleeve gastrectomy as a definitive surgical procedure for morbid obesity. mid-term results. Obes Surg. 2013; 23: 292-299.

14. Asztalos BF, Swarbrick MM, Schaefer EJ, Dallal GE, Horvath KV, Ai M, et al. Effects of weight loss, induced by gastric bypass surgery, on HDL remodeling in obese women. J Lipid Res. 2010; 51: 2405-2412.

15. Zubiaga L, Ruiz-Tovar J, Giner L, Gonzalez J, Aguilar M, García A, et al. Valoración del riesgo cardiovascular después de gastrectomía vertical: comparativa del IMC, la adiposidad, el índice de Framingham y el índice aterogénico como marcadores del éxito de la cirugía. Nutr Hosp 2016; 33: 832-837.

16. Leyba JL, Llopis SN, Aulestia SN. Laparoscopic Roux-en-Y gastric bypass versus laparoscopic sleeve gastrectomy for the treatment of morbid obesity. a prospective study with 5 years of follow-up. Obes Surg. 2014; 24: 2094-2098.

17. Molina-Ayala M, Rodríguez-González A, AlbarránSánchez A, Ferreira-Hermosillo A, Ramírez-Rentería C, Luque-de León E, et al. Características clínicas y bioquímicas de pacientes con obesidad extrema al ingreso y un año después de someterse a cirugía bariátrica. Rev Med Inst Mex Seguro Soc. 2016; 54: 5118-5125.

18. Reyes-Pérez A, Sánchez-Aguilar H, VelázquezFernández D, Rodríguez-Ortíz D, Mosti M, Herrera MF. Analysis of causes and risk factors for hospital readmission after Roux-en-Y Gastric Bypass. Obes Surg. 2016; 26: 257.
19. Romero-Ibarguengoitia ME, Lerman-Garber I, HerreraHernández MF, Pablo-Pantoja J, Sierra- Salazar M, Lopez-Rosales $F$, et al. Bypass gástrico laparoscópico en $\mathrm{Y}$ de Roux y obesidad mórbida. Experiencia en el Instituto Nacional de Ciencias Médicas y Nutrición "Salvador Zubirán". Rev Invest Clin. 2009; 61: 186193.

20. Sjostrom L, Peltonen $M$, Jacobson P, Sjostrom CD, Karason K, Wedel H, et al. Bariatric surgery and longterm cardiovascular events. JAMA. 2012; 307: 56-65.

Consideraciones y responsabilidad ética: Privacidad de los datos. De acuerdo a los protocolos establecidos en el centro de trabajo de los autores, éstos declaran que han seguido los protocolos sobre la privacidad de datos de pacientes preservando su anonimato.

Financiamiento: No se recibió apoyo financiero para la realización de este trabajo.

Conflicto de intereses: Los autores declaran que no existe ningún conflicto de intereses en la realización del trabajo.

Correspondencia:

Dra. Gabriela Ruiz-Mar

Dr. Balmis Núm. 148,

Col. Doctores, 06720,

Alcaldía Cuauhtémoc,

Ciudad de México, CDMX.

Tel: 55 5458-8306

E-mail: rvgaby@hotmail.com 